\title{
APPROXIMATION NUMBERS OF MATRIX TRANSFORMATIONS AND INCLUSION MAPS
}

\author{
M. GUPTA AND L. R. ACHARYA
}

\begin{abstract}
In this paper we establish relationships of the approximation numbers of matrix transformations acting between the vector-valued sequence spaces spaces of the type $\lambda(X)$ defined corresponding to a scalar-valued sequence space $\lambda$ and a Banach space $(X,\|\cdot\|)$ as

$$
\lambda(X)=\left\{\bar{x}=\left\{x_{i}\right\}: x_{i} \in X, \forall i \in \mathbb{N},\left\{\left\|x_{i}\right\|_{X}\right\} \in \lambda\right\}
$$

with those of their component operators. This study leads to a characterization of a diagonal operator to be approximable. Further, we compute the approximation numbers of inclusion maps acting between $\ell^{p}(X)$ spaces for $1 \leq p \leq \infty$.
\end{abstract}

\section{Introduction}

Eversince the inception of approximation numbers of operators on Banach spaces in 1963 by A. Pietsch [12], mathematicians have been interested in finding the estimates of these numbers for various embedding maps between function spaces, sequence spaces etc., for instance one may refer to [7, 8, 10, 17]. However, motivated by the work of Hutton [9], we estimate these numbers for inclusion mappings between vector valued sequence spaces.

Throughout this paper we denote by $X, Y$ and $Z$ the Banach spaces defined over the complex field $\mathbb{C}$ and by $U_{X}$, the closed unit ball in the space $X . \mathscr{L}(X, Y)$ represents the class of all bounded linear operators from $X$ to $Y . \mathbb{N}$ stands for the set of all natural numbers.

For $T \in \mathscr{L}(X, Y)$ and $n \in \mathbb{N}$, the $n^{\text {th }}$ approximation number of $T$ is given by

$$
a_{n}(T)=\inf \{\|T-A\|: A \in \mathscr{L}(X, Y), \operatorname{rank}(A)<n\}
$$

and $T$ is said to be approximable if $a_{n}(T) \rightarrow 0$ as $n \rightarrow \infty$. The approximation numbers satisfy the following algebraic properties for well defined addition and composition of operators $R, S$ and $T$.

$$
\text { 1. }\|S\|=a_{1}(S) \geq a_{2}(S) \geq \cdots \geq a_{n}(S) \geq \cdots \geq 0 ;
$$

Corresponding author: M. Gupta.

2000 Mathematics Subject Classification. 47B06, 47B37, 46B45.

Key words and phrases. Approximation numbers, matrix transformations, sequence spaces. 
2. $a_{n}(S+T) \leq\|S\|+a_{n}(T)$;

3. $a_{n}(R S T) \leq\|R\| a_{n}(S)\|T\|$;

4. If $\operatorname{rank}(S)<n$, then $a_{n}(S)=0$;

5. $a_{n}\left(I_{X}\right)=1$, whenever $\operatorname{dim}(X) \geq n$, where $I_{X}$ is the identity mapping on the Banach space $X$.

A function $s$ associating each linear operator $T$ with a sequence $\left\{s_{n}(T)\right\}$ of non-negative reals satisfying the properties analogous to [1]-[5] has been termed in the literature as an $s$ function. The map defined by the approximation numbers is indeed the largest $s$-function $[14,15]$. For various results on $s$-numbers as well as approximation numbers, we refer to $[2,9,13]$ and [16]. As evident from the definition, approximation numbers are the measure of nearness of an operator $T \in \mathscr{L}(X, Y)$ by finite rank operators, it is therefore natural to ask whether the compactness of $T$ has any connection with the rate of decrease of these numbers. Indeed, every approximable operator is compact but converse may not be true as shown by Enflo in [3].

Coming to the study of domain and range spaces of the matrix transformations to be studied in this paper, we refer to $[4,5]$ and [11], for detailed theory of these spaces, namely the vector valued sequence spaces. We denote by $\Omega(X)$, the class of all sequences from $X$ and by $\Phi(X)$, the subspace of $\Omega(X)$ consisting of all finitely non-zero sequences. A vector valued sequence space $\Lambda(X)$ is a subspace of $\Omega(X)$ containing $\Phi(X)$. The $k^{\text {th }}$ section of $\bar{x}=\left\{x_{i}\right\}$ is the sequence $\bar{x}^{(k)}=\left\{x_{1}, x_{2}, \ldots, x_{k}, 0,0, \ldots\right\}$. For $x \in X, \delta_{i}^{x}$ denotes the sequence $\{0,0, \ldots, 0, x, 0, \ldots\}$, where $x$ is placed at the $i^{t h}$ co-ordinate. In case $X=\mathbb{C}$, we write $\omega$ for $\Omega(X), \phi$ for $\Phi(X), \lambda$ for $\Lambda(X)$ and we denote by $e^{i}$ the element $\delta_{i}^{1}$ of $\lambda$. A linear map $Z: \Lambda(X) \rightarrow \Lambda(Y)$ is said to be a matrix transformation, if there exists a matrix $\left[Z_{i j}\right]$ of linear maps, $Z_{i j}: X \rightarrow Y$ for each $i, j \in \mathbb{N}$, such that for every $\bar{x}=\left\{x_{n}\right\}$ in $\Lambda(X)$, the series $\sum_{j=1}^{\infty} Z_{i j}\left(x_{j}\right)$ converges to some element $y_{i} \in Y, \forall i \in \mathbb{N}$ and $\left\{y_{i}\right\} \in \Lambda(Y)$ i.e.

$$
y_{i}=\sum_{j=1}^{\infty} Z_{i j}\left(x_{j}\right)=P_{i, \Lambda(Y)}(Z(\bar{x}))
$$

where $P_{i, \Lambda(Y)}: \Lambda(Y) \rightarrow Y$ is defined as

$$
P_{i, \Lambda(Y)}(\bar{y})=y_{i}, \quad \forall i \in \mathbb{N} \text { and } \forall \bar{y}=\left\{y_{i}\right\} \in \Lambda(Y) \text {. }
$$

If in the above definition $Z_{i j} \equiv 0, \forall i \neq j$, then $Z$ is called a diagonal operator. A subset $M$ of $\Lambda(X)$ is said to be normal if for any $\bar{x}=\left\{x_{i}\right\} \in M$ and $\alpha_{i} \in \mathbb{K}$, with $\left|\alpha_{i}\right| \leq 1, i \geq 1$, the sequence $\left\{\alpha_{i} x_{i}\right\} \in M$. A vector-valued sequence space $\Lambda(X)$ equipped with a Hausdorff locally convex topology $\mathscr{F}$ is called ( $i) a$ GK-space if the maps $P_{n, \Lambda(X)}: \Lambda(X) \rightarrow X, P_{n, \Lambda(X)}(\bar{x})=x_{n}$, 
for each $n \geq 1$, are continuous; ( $i$ i ) a GAK-space if $\Lambda(X)$ is a GK-space and for each $\bar{x}=\left\{x_{i}\right\}$ from $\Lambda(X), \bar{x}^{(n)} \rightarrow \bar{x}$ as $n \rightarrow \infty$, in $\mathscr{F}$. In the case when $X=\mathbb{K}$, the space in definitions $(i)$ and ( $i$ i ) is referred to as a $K$-space and an $A K$-space. A norm $\|\cdot\|_{\lambda}$ of a scalar valued sequence space $\lambda$ is said to be monotone if $\left|\alpha_{i}\right| \leq\left|\beta_{i}\right|, \forall i \in \mathbb{N}$ implies $\left\|\left\{\alpha_{i}\right\}\right\|_{\lambda} \leq\left\|\left\{\beta_{i}\right\}\right\|_{\lambda}$.

The particular types of vector-valued sequence spaces which we consider in this paper are

$$
\lambda(X)=\left\{\bar{x}=\left\{x_{i}\right\}: x_{i} \in X, \forall i \in \mathbb{N} \text { and }\left\{\left\|x_{i}\right\|_{X}\right\} \in \lambda\right\},
$$

where $\left(\lambda,\|\cdot\|_{\lambda}\right)$ is a scalar valued Banach sequence space. $\lambda(X)$ is a Banach space with the norm given by

$$
\|\bar{x}\|_{\lambda(X)}=\left\|\left\{\left\|x_{i}\right\|_{X}\right\}\right\|_{\lambda}
$$

for any $\bar{x}=\left\{x_{i}\right\} \in \lambda(X),[4][6]$.

\section{Approximation numbers of matrix transformations from $\lambda(X)$ to $\mu(Y)$}

Let us recall from our earlier work [1] a few results and notations concerning the matrix transformations from $\lambda(X)$ to $\mu(Y)$, where we assume that $\lambda$ and $\mu$ are any two normal, normed scalar valued sequence spaces containing $\phi$, which are equipped with the monotone norms $\|.\|_{\lambda}$ and $\|.\|_{\mu}$ respectively. Further, $\mu$ is an AK-space and $\left\|e^{i}\right\|_{\lambda}=\left\|e^{i}\right\|_{\mu}=1, \forall i \in \mathbb{N}$. It is shown in [1] that if $Z=\left[Z_{i j}\right]$ is a matrix transformation from $\lambda(X)$ to $\mu(Y)$ with $\left\{\sum_{j=1}^{\infty}\left\|Z_{i j}\right\|\right\}_{i=1}^{\infty}$ $\epsilon \mu$, then $Z$ is a bounded linear operator from $\lambda(X)$ to $\mu(Y)$ satisfying

$$
\sup _{i, j}\left\|Z_{i j}\right\| \leq\|Z\| \leq\left\|\left\{\sum_{j=1}^{\infty}\left\|Z_{i j}\right\|\right\}_{i=1}^{\infty}\right\|_{\mu} .
$$

It has also been noted in [1] that the diagonal operators $Z$ from $\lambda(X)$ into $\mu(Y)$ are in fact the maps from $\lambda(X)$ into $\lambda(Y)$ and in this case $\|Z\|=\sup _{i}\left\|Z_{i i}\right\|$.

For $k \in \mathbb{N}$, let us write

$$
\lambda_{k}(X)=X \times X \times \cdots \times X(k \text { times })
$$

We equip $\lambda_{k}(X)$ with the norm given by

$$
\left\|\left(x_{1}, x_{2}, \ldots, x_{k}\right)\right\|_{\lambda_{k}(X)}=\left\|\left\{z_{i}\right\}\right\|_{\lambda(X)} ;
$$

where $z_{i}=x_{i}, 1 \leq i \leq k$ and $z_{i}=0, \forall i>k$.

Corresponding to a matrix transformation $Z$ let us define $Z^{k}$ and $Z_{k}$ as the linear operators from $\lambda(X)$ to $\mu_{k}(Y)$ and to $\mu(Y)$ respectively, given by

$$
Z^{k}(\bar{x})=\left(\sum_{j=1}^{\infty} Z_{1 j} x_{j}, \sum_{j=1}^{\infty} Z_{2 j} x_{j}, \ldots, \sum_{j=1}^{\infty} Z_{k j} x_{j}\right)
$$


and

$$
Z_{k}(\bar{x})=\left\{\sum_{j=1}^{\infty} Z_{1 j} x_{j}, \sum_{j=1}^{\infty} Z_{2 j} x_{j}, \ldots, \sum_{j=1}^{\infty} Z_{k j} x_{j}, 0,0 \ldots\right\}
$$

for $\bar{x}=\left\{x_{i}\right\} \in \lambda(X)$.

For each $k \in \mathbb{N}$, we also consider the projection and inclusion maps $P_{k, \lambda(X)}: \lambda(X) \rightarrow X$, $P_{\lambda(X)}^{k}: \lambda(X) \rightarrow \lambda_{k}(X), I_{k, \lambda(X)}: X \rightarrow \lambda(X)$ and $I_{\lambda(X)}^{k}: \lambda_{k}(X) \rightarrow \lambda(X)$, defined as

$$
\begin{aligned}
& P_{k, \lambda(X)}(\bar{x})=x_{k}, \bar{x}=\left\{x_{k}\right\} \in \lambda(X) ; \\
& P_{\lambda(X)}^{k}(\bar{x})=\left(x_{1}, x_{2}, \ldots, x_{k}\right), \bar{x}=\left\{x_{k}\right\} \in \lambda(X) ; \\
& I_{k, \lambda(X)}(x)=\delta_{k}^{x}, \quad x \in X
\end{aligned}
$$

and

$$
I_{\lambda(X)}^{k}\left(x_{1}, x_{2}, \ldots, x_{k}\right)=\left\{x_{1}, x_{2}, \ldots, x_{k}, 0,0, \ldots\right\}, \quad\left(x_{1}, x_{2}, \ldots, x_{k}\right) \in \lambda_{k}(X) .
$$

Note that the norm of any of the maps defined above can not exceed one. Assuming that $Z: \lambda(X) \rightarrow \mu(Y)$ is a matrix transformation with $\left\{\sum_{j=1}^{\infty}\left\|Z_{i j}\right\|\right\}_{i=1}^{\infty} \in \mu$, we begin with

Proposition 2.1. For a fixed $k \in \mathbb{N}$,

$$
a_{n}\left(Z^{k}\right)=a_{n}\left(Z_{k}\right), \forall n \in \mathbb{N} \text {. }
$$

Proof. Note that $Z^{k}=P_{\mu(Y)}^{k} \cdot Z_{k}$ and $Z_{k}=I_{\mu(Y)}^{k} \cdot Z^{k}$. The result now follows from the multiclicative property of approxmation numbers.

Proposition 2.2. For a fixed $k \in \mathbb{N}$,

$$
a_{n}\left(Z_{k}\right) \leq a_{n}(Z), \forall n \in \mathbb{N} \text {. }
$$

Proof. Since $Z_{k}=I_{\mu(Y)}^{k} \cdot P_{\mu(Y)}^{k} \cdot Z$, applying the multiplicative property of approximation numbers, the required inequality follows.

Corollary 2.3. For each $n \in \mathbb{N}$,

$$
a_{n}(Z)=\lim _{k \rightarrow \infty} a_{n}\left(Z_{k}\right)
$$

Proof. To get this result, use additive property of approximation numbers to get the following inequality

$$
0 \leq a_{n}(Z)-a_{n}\left(Z_{k}\right) \leq\left\|\left(0, \ldots, 0, \sum_{j=1}^{\infty}\left\|Z_{(k+1) j}\right\|, \sum_{j=1}^{\infty}\left\|Z_{(k+2) j}\right\|, \ldots\right)\right\|_{\mu},
$$

for any $n, k \in \mathbb{N}$ and the AK-ness of $\mu$.

However, the validity of the above result doesn't yield $\left\{\sum_{j=1}^{\infty}\left\|Z_{i j}\right\|\right\}_{i=1}^{\infty} \in \mu$ or the AK-ness of $\mu$, as illustrated in 
Example 2.4. If $Z$ is the identity mapping on $\ell^{p}, 1 \leq p \leq \infty$, then we have

$$
a_{n}(Z)=\lim _{k \rightarrow \infty} a_{n}\left(Z_{k}\right)
$$

But $\ell^{\infty}$ is not an AK-space and $\left\{\sum_{j=1}^{\infty}\left\|Z_{i j}\right\|\right\}_{i=1}^{\infty}$ is not in $\ell^{p}$ for $1 \leq p<\infty$.

Theorem 2.5. For each $n \in \mathbb{N}, a_{n}\left(Z_{i j}\right) \leq a_{n}(Z), \forall i, j \in \mathbb{N}$.

Proof. Since $Z_{i j}=P_{i, \mu(Y)} \cdot Z \cdot I_{j, \lambda(X)}$, the result follows from the multiplicative property of approximation numbers.

The above result immediately leads to

Corollary 2.6. If $Z=\left[Z_{i j}\right]$ from $\lambda(X)$ to $\mu(Y)$ is an approximable matrix transformation then $Z_{i j}: X \rightarrow Y$ is approximable (hence compact), for each $i, j \in \mathbb{N}$.

The converse of Theorem 2.5 holds in the following form-

Theorem 2.7. For fixed $k \in \mathbb{N}$,

$$
a_{k n}\left(Z^{k}\right) \leq\left\|e^{(k)}\right\|_{\mu} \max _{1 \leq i \leq k}\left\{a_{n}\left(Z_{i i}\right)+\sum_{j \neq i}\left\|Z_{i j}\right\|\right\}, n \in \mathbb{N} ;
$$

where $e^{(k)}$ represents the $k^{\text {th }}$ section of $e=\{1,1, \ldots, 1, \ldots\}$.

Proof. First we note that $\sum_{j=1}^{\infty}\left\|Z_{i j}\right\|<\infty$, for each $i \in \mathbb{N}$, since the sequence $\left\{\sum_{j=1}^{\infty}\left\|Z_{i j}\right\|\right\}_{i=1}^{\infty} \in$ $\mu \subseteq \ell^{\infty}$. Now $\forall \epsilon>0$, we can find an operator $A_{i j} \in \mathscr{L}(X, Y)$ of rank $m, m<n$ such that

$$
\left\|Z_{i j}-A_{i j}\right\| \leq a_{n}\left(Z_{i j}\right)+\epsilon
$$

For any $\bar{x}=\left\{x_{i}\right\} \in \lambda(X)$, define

$$
A^{k}(\bar{x})=\left(A_{11}\left(x_{1}\right), \ldots, A_{k k}\left(x_{k}\right)\right) .
$$

Then $\operatorname{rank}\left(A^{k}\right)<k n$ and so

$$
a_{k n}\left(Z^{k}\right) \leq\left\|Z^{k}-A^{k}\right\| \leq\left\|e^{(k)}\right\|_{\mu} \max _{1 \leq i \leq k}\left\{a_{n}\left(Z_{i i}\right)+\sum_{j \neq i}\left\|Z_{i j}\right\|\right\}, n \in \mathbb{N} .
$$

Indeed, $\forall \bar{x}=\left\{x_{i}\right\} \in U_{\lambda(X)}$ we have

$$
\left\|\left(Z^{k}-A^{k}\right)(\bar{x})\right\|_{\mu_{k}(Y)} \leq\left\|e^{(k)}\right\|_{\mu} \max _{1 \leq i \leq k}\left\{\left\|Z_{i i}\left(x_{i}\right)-A_{i i}\left(x_{i}\right)\right\|_{Y}+\sum_{j \neq i}\left\|Z_{i j}\right\|\right\} .
$$


Corollary 2.8. If $\left\{\alpha_{i}\right\} \in \mu$ is such that $\alpha_{i}=0,1 \leq i \leq k$ and $\alpha_{i}=\sum_{j=1}^{\infty}\left\|Z_{i j}\right\|$, for each $i>k$, then

$$
a_{k n}(Z) \leq\left\|\left\{\alpha_{i}\right\}\right\|_{\mu}+\left\|e^{(k)}\right\|_{\mu} \max _{1 \leq i \leq k}\left\{a_{n}\left(Z_{i i}\right)+\sum_{j \neq i}\left\|Z_{i j}\right\|\right\} .
$$

Remark 2.9. In case of a diagonal operator $Z$, the above inequality reduces to the following form

$$
a_{k n}(Z) \leq \sup _{i=k+1}^{\infty}\left\|Z_{i i}\right\|+\left\|e^{(k)}\right\|_{\mu} \max _{i=1}^{k}\left(a_{n}\left(Z_{i i}\right)\right)
$$

Now we prove

Theorem 2.10. The diagonal operator $Z: \lambda(X) \rightarrow \lambda(Y)$ is approximable if and only if $Z_{i i}$ is approximable, for each $i \in \mathbb{N}$ and $\left\|Z_{i i}\right\| \rightarrow 0$ as $i \rightarrow \infty$.

Proof. It is clear from Theorem 2.5 that each $Z_{i i}, i \in \mathbb{N}$, is approximable if $Z$ is approximable. Further note that for any $\epsilon>0$, we can find $\left\{x_{i}\right\} \subseteq U_{X}$ such that

$$
\left\|Z_{i i}\right\|<\left\|Z_{i i}\left(x_{i}\right)\right\|_{Y}+\frac{\epsilon}{2} .
$$

Since $Z$ is compact (being approximable), we can find $m_{1}, m_{2}, \ldots, m_{n} \in \mathbb{N}$ such that for every $i \in \mathbb{N}$ we have

$$
\left\|Z\left(\delta_{i}^{x_{i}}\right)-Z\left(\delta_{m_{k}}^{x_{m_{k}}}\right)\right\|_{\lambda(Y)}<\frac{\epsilon}{2}
$$

for some $1 \leq k \leq n$.

By choosing $N_{0}=\max \left\{m_{1}, m_{2}, \ldots, m_{n}\right\}$ and using the monotonicity of $\|\cdot\|_{\lambda}$ we get

$$
\left\|Z_{i i}\left(x_{i}\right)\right\|_{Y} \leq\left\|Z\left(\delta_{i}^{x_{i}}\right)-Z\left(\delta_{m_{k}}^{x_{m_{k}}}\right)\right\|_{\lambda(Y)}<\frac{\epsilon}{2}, \forall i>N_{0} .
$$

Hence $\left\|Z_{i i}\right\| \rightarrow 0$ as $i \rightarrow \infty$. For the converse note that for any given $\epsilon>0$, there exists a $k_{o} \in \mathbb{N}$ such that

$$
\sup _{i=k_{o}+1}^{\infty}\left\|Z_{i i}\right\| \leq \epsilon / 2
$$

and approximability of $Z_{i i}$ 's imply that there exists $n_{o} \in \mathbb{N}$ depending upon $k_{o}$ such that

$$
a_{n}\left(Z_{i i}\right) \leq \epsilon /\left(2\left\|e^{\left(k_{o}\right)}\right\|_{\mu}\right), \forall i=1,2, \ldots, k_{o}, \forall n \geq n_{o} .
$$

Hence, from Remark 2.9, we get $a_{n_{o} k_{o}}(Z) \leq \epsilon$. Thus

$$
a_{j}(Z) \leq \epsilon, \forall j \geq n_{o} k_{o}, j \in \mathbb{N} \text {. }
$$

$\Rightarrow Z$ is approximable. 


\section{Approximation numbers of inclusion maps}

In this section we compute the approximation numbers of the inclusion maps between spaces of the type $\ell^{p}(X), 1 \leq p \leq \infty$, using the results proved in the preceding section and thus conclude that these maps are not approximable. Besides generalizing the results to vectorvalued $\ell^{p}(X)$ spaces, we prove in the following result how the dimension of the underlying space affects the value of the approximation number of the inclusion map. Indeed, we prove

Theorem 3.1. For the inclusion map $I: \ell^{1}(X) \rightarrow \ell^{\infty}(X)$,

$$
\frac{1}{2} \leq a_{n}(I) \leq 1, \forall n \geq 2, n \in \mathbb{N} .
$$

Further, $a_{n}(I)=1, \forall n \in \mathbb{N}$, in case $X$ is an infinite dimensional Banach space. If $\operatorname{dim}(X)=k$, we have $a_{n}(I)=1,1 \leq n \leq k$ and $a_{n}(I)=\frac{1}{2}, \forall n>k$.

Proof. Since $a_{1}(I)=\|I\|=1$, we have $a_{n}(I) \leq 1, \forall n \in \mathbb{N}$.

If there exists $n \in \mathbb{N}, n \geq 2$ with $a_{n}(I)<\frac{1}{2}$, choose $\epsilon>0$ such that

$$
a_{n}(I)<\frac{1}{2}-\epsilon .
$$

For this $\epsilon>0$, there exists $A \in \mathscr{L}\left(\ell^{1}(X), \ell^{\infty}(X)\right)$ with $\operatorname{rank}(A)<n$ such that

$$
\|I-A\|<a_{n}(I)+\frac{\epsilon}{2}<\frac{1}{2}-\frac{\epsilon}{2} .
$$

We now fix $x \in X$ with $\|x\|=1$ and let $A\left(\delta_{i}^{x}\right)=\left\{y_{i j}^{x}\right\}, \forall i \in \mathbb{N}$.

Then from (3.2), for each $i \in \mathbb{N}$, we get

$$
\left.\max _{j \neq i} \sup _{j \neq i}\left\|y_{i j}^{x}\right\|_{X},\left\|x-y_{i i}^{x}\right\|_{X}\right\}<\frac{1}{2}-\frac{\epsilon}{2}
$$

Since $A$ is a finite rank operator, the set

$$
\mathscr{S}^{A}=\left\{\bar{y} \in A\left(\ell^{1}(X)\right):\|\bar{y}\|_{\ell^{\infty}(X)} \leq\|A\|\right\}
$$

is relatively compact. Also note that for each $i \in \mathbb{N}$, the element $A\left(\delta_{i}^{x}\right) \in \mathscr{S}^{A}$. Hence for a fixed $x \in X$ with $\|x\|_{X}=1$ and $i, j \in \mathbb{N}$ with $i \neq j$ we have

$$
\left\|A\left(\delta_{i}^{x}\right)-A\left(\delta_{j}^{x}\right)\right\|_{\ell^{\infty}(X)} \geq\left\|y_{i k}^{x}-y_{j k}^{x}\right\|_{X},
$$

for each $k \in \mathbb{N}$.

In particular when $i=k$, we get

$$
\left\|A\left(\delta_{i}^{x}\right)-A\left(\delta_{j}^{x}\right)\right\|_{\ell^{\infty}(X)} \geq\left\|y_{i i}^{x}-y_{j i}^{x}\right\|_{X} \geq\left\|y_{i i}^{x}\right\|_{X}-\left\|y_{j i}^{x}\right\|_{X} .
$$


Since $\left\|x-y_{i i}^{x}\right\|_{X}<\frac{1}{2}-\frac{\epsilon}{2}$ and $\left\|y_{j i}^{x}\right\|_{X}<\frac{1}{2}-\frac{\epsilon}{2}$, from (3.3) we get,

$$
\left\|A\left(\delta_{i}^{x}\right)-A\left(\delta_{j}^{x}\right)\right\|_{\ell^{\infty}(X)}>\|x\|_{X}-\frac{1}{2}+\frac{\epsilon}{2}-\frac{1}{2}+\frac{\epsilon}{2}=\epsilon>0,
$$

for each $i \neq j$. This contradicts the fact that $\mathscr{S}^{A}$ is relatively compact and hence (3.1) holds.

Since each component operator of $I$ is the identity operator on $X$, using the Property [5] of approximation numbers and Theorem 2.5, we conclude that $a_{n}(I)=1, \forall n \in \mathbb{N}$, in case $X$ is infinite dimensional space.

If $\operatorname{dim}(X)=k$, let $\left\{u_{1}, u_{2}, \ldots, u_{k}\right\}$ be a basis of $X$. If $x \in X$ is such that $x=\sum_{i=1}^{k} \alpha_{j} u_{j}$, we assume that the norm on $X$ is given by $\|x\|_{X}=\sum_{i=1}^{k}\left|\alpha_{j}\right|$. Note that $\left\|u_{j}\right\|=1, \forall j=1,2, \ldots, k$. Let us take $\bar{x}=\left\{x_{i}\right\} \in \ell^{1}(X)$, where $x_{i}=\sum_{j=1}^{k} \alpha_{i j} u_{j}$. Since $\sum_{i=1}^{\infty}\left\|u_{j}\right\|_{X}<\infty$, we get $\sum_{i=1}^{\infty} \sum_{j=1}^{k}\left|\alpha_{i j}\right|=$ $M<\infty$. For $1 \leq j \leq k$, define

$$
\beta_{j}=\frac{1}{2} \sum_{i=1}^{\infty} \alpha_{i j}
$$

Then $\beta_{j}$ is well defined and

$$
\sum_{j=1}^{k}\left|\beta_{j}\right|<\sum_{j=1}^{k} \sum_{i=1}^{\infty}\left|\alpha_{i j}\right|<\infty .
$$

Write $z=\sum_{j=1}^{k} \beta_{j} u_{j}$ and $\bar{z}=\{z, z, z, \ldots\}$. Then

$$
\|\bar{z}\|_{\ell^{\infty}(X)}=\|z\|_{X}=\sum_{j=1}^{k}\left|\beta_{j}\right| \leq \frac{1}{2} M<\infty .
$$

$\Rightarrow \bar{z} \in \ell^{\infty}(X)$. Define $A: \ell^{1}(X) \rightarrow \ell^{\infty}(X)$ as

$$
A(\bar{x})=\{z, z, \ldots, z, \ldots\}
$$

Then, $\operatorname{rank}(A)=k$; indeed the elements $\bar{u}_{j}=\left(u_{j}, u_{j}, \ldots, u_{j}, \ldots\right), 1 \leq j \leq k$, would span the range of $A$. Also, for any $\bar{x}=\left\{x_{i}\right\} \in \ell^{1}(X)$ with $\|\bar{x}\|_{\ell^{1}(X)}=1$, we have $\|(I-A) \bar{x}\|_{\ell^{\infty}(X)}=\sup _{i=1}^{\infty} \| x_{i}-$ $z \|_{X}$. If $x_{i}$ is given by $\sum_{j=1}^{k} \alpha_{i j} u_{j}$, for each $i \in \mathbb{N}$, we have

$$
\sup _{i=1}^{\infty}\left\|x_{i}-z\right\|_{X} \leq \frac{1}{2} \sum_{i=1}^{\infty} \sum_{j=1}^{k}\left|\alpha_{i j}\right|=\frac{1}{2} .
$$

$\Rightarrow\|I-A\| \leq \frac{1}{2}$. Hence $a_{n}(I)=\frac{1}{2}, \forall n>k$.

To show that $a_{n}(I)=1,1 \leq n \leq k$, note that $a_{1}(I)=1$. If $1<n \leq k$, we can conclude that $a_{k}(I)=1$, making use of Property [5] of approximation numbers, the fact that each component operator of $I$ is the identity operator on $X$ and Theorem 2.5. Since approximation numbers are decreasing in nature, we get the required result. 
Remark 3.2. One can prove this result with respect to any norm on a finite dimensional normed linear space $\mathrm{X}$ of dimension $k$, which is monotone in the sense of its co-ordinate representation with respect to a basis $\left\{u_{1}, u_{2}, \ldots, u_{k}\right\}$ such that $\left\|u_{i}\right\|_{X}=1$, for each $i=1,2, \ldots, k$.

Theorem 3.3. For the inclusion map $I: \ell^{p}(X) \rightarrow \ell^{\infty}(X), 1<p \leq \infty$,

$$
a_{n}(I)=1, \forall n \in \mathbb{N} \text {. }
$$

Proof. When $p=\infty$, the map under consideration is the identity map on $\ell^{\infty}(X)$ and in this case we get $a_{n}(I)=1, \forall n \in \mathbb{N}$ using the Property [5] of approximation numbers. For $1<p<$ $\infty$, we always have $a_{n}(I) \leq 1$.

If $a_{n}(I)<1$, for some $n \in \mathbb{N}$, then we can find $\epsilon>0$ such that $a_{n}(I)<1-\epsilon$. For this $\epsilon>0$, there exists $A \in \mathscr{L}\left(\ell^{p}(X), \ell^{\infty}(X)\right)$ of rank $m<n$ such that

$$
\|I-A\|<1-\frac{\epsilon}{2}
$$

Note that $A$ can be expressed as $A(\bar{x})=\sum_{i=1}^{m} f_{i}(\bar{x}) \bar{y}_{i}, \forall \bar{x} \in \ell^{1}(X)$, where $f_{i}=\left\{f_{i j}\right\} \in\left[\ell^{p}(X)\right]^{*}=$ $\ell^{q}\left(X^{*}\right)$ (cf. [5][11]) and $\bar{y}_{i}=\left\{y_{i j}\right\} \in \ell^{\infty}(X)$, for each $i=1,2, \ldots, m$.

Let us fix $x \in X$ with $\|x\|_{X}=1$. We then have

$$
\sum_{j \geq 1}\left|f_{i j}(x)\right|^{q} \leq \sum_{j \geq 1}\left\|f_{i j}\right\|^{q}, \quad \forall i=1,2, \ldots m .
$$

From equation (3.4) we get

$$
\left\|x-\sum_{i=1}^{m} f_{i}\left(\delta_{j}^{x}\right) y_{i j}\right\|_{X} \leq\left\|(I-A) \delta_{j}^{x}\right\|_{\ell^{\infty}(X)}<1-\frac{\epsilon}{2}, \forall j \in \mathbb{N} .
$$

Let $M=\max _{1 \leq i \leq m}\left\|\bar{y}_{i}\right\|_{\ell^{\infty}(X)}$. Note that $M>0$. From (3.5) we get

$$
\frac{\epsilon}{2}<\left\|\sum_{i=1}^{m} f_{i}\left(\delta_{j}^{x}\right) y_{i j}\right\|_{X} \leq M \cdot \sum_{i=1}^{m}\left|f_{i}\left(\delta_{j}^{x}\right)\right| .
$$

Hence we have

$$
\sum_{i=1}^{m}\left|f_{i}\left(\delta_{j}^{x}\right)\right|=\sum_{i=1}^{m}\left|f_{i j}(x)\right|>\frac{\epsilon}{2 M}, \forall j \in \mathbb{N} .
$$

On the other hand, there is $j_{o} \in \mathbb{N}$ such that

$$
\sum_{j \geq j_{o}}\left|f_{i j}(x)\right|^{q}<\left(\frac{\epsilon}{2 m M}\right)^{q},
$$

for each $i=1,2, \ldots m$. Hence

$$
\sum_{i=1}^{m}\left|f_{i j_{o}}(x)\right|<\frac{\epsilon}{2 M} .
$$

This is a contradiction to (3.6). Thus the result holds. 
Theorem 3.4. For $1 \leq p \leq q<\infty$, if $I: \ell^{p}(X) \rightarrow \ell^{q}(X)$, is the natural injection, then $a_{n}(I)=$ $1, \forall n \in \mathbb{N}$.

Proof. We consider two cases for the proof of the result.

Case 1: Let $1<p \leq q<\infty$. Denote by $I_{r}$ the natural injection from $\ell^{r}(X)$ to $\ell^{\infty}(X)$. Then $I_{p}=I_{q} \cdot I$. Hence $a_{n}(I) \geq 1$. Since $a_{n}(I) \leq a_{1}(I)=1, \forall n \in \mathbb{N}$, result is proved in this case.

Case 2: Let $p=1$. Assume $a_{n}(I)<1$ for some $n \in \mathbb{N}$. Then we can find $\epsilon>0$ and $A: \ell^{1}(X) \rightarrow$ $\ell^{q}(X)$ with $\operatorname{rank}(A)<n$ such that

$$
\|I-A\|<1-\frac{\epsilon}{2}
$$

Let $\mathscr{S}^{A}=\left\{\bar{x} \in A\left(\ell^{1}(X)\right):\|\bar{x}\|_{\ell^{q}(X)} \leq\|A\|\right\}$. Note that $A\left(\delta_{i}^{x}\right) \in \mathscr{S}^{A}, \forall i \in \mathbb{N}$ and $x \in X$ with $\|x\|_{X}=1$. Since $\mathscr{S}^{A}$ is relatively compact, choose $\frac{\varepsilon}{6}$-net $\left\{\bar{y}_{k}=\left\{y_{k j}\right\}: k=1,2, \ldots, m\right\}$ for $\mathscr{S}^{A}$. We can now find a $j_{o} \in \mathbb{N}$ such that

$$
\left(\sum_{j \geq j_{o}}\left\|y_{k j}\right\|_{X}^{q}\right)^{1 / q}<\epsilon / 6, \quad \forall k=1,2, \ldots, m
$$

For a fixed $x \in X$ with $\|x\|_{X}=1$ and each $i \in \mathbb{N}$ there exists $1 \leq m_{i} \leq m$ such that

$$
\left\|A\left(\delta_{i}^{x}\right)-\bar{y}_{m_{i}}\right\|_{\ell q(X)}<\epsilon / 6 .
$$

Let $A\left(\delta_{i}^{x}\right)=\left\{a_{i j}^{x}\right\}$ for each $i \in \mathbb{N}$. Then

$$
\left(\sum_{j \geq 1}\left\|a_{i j}^{x}-y_{m_{i} j}\right\|_{X}^{q}\right)^{1 / q}<\epsilon / 6
$$

Using Minkowski's inequality, from (3.7) and (3.8) we get

$$
\left(\sum_{j \geq j_{o}}\left\|a_{i j}^{x}\right\|_{X}^{q}\right)^{1 / q}<\epsilon / 3, \forall i \in \mathbb{N} \Rightarrow\left\|a_{i j}^{x}\right\|_{X}<\epsilon / 3, \forall j \geq j_{o} .
$$

Also, for each $i \in \mathbb{N}$

$$
\begin{aligned}
& \left\{\left\|x-a_{i i}^{x}\right\|_{X}^{q}+\sum_{j \neq i}\left\|a_{i j}^{x}\right\|_{X}^{q}\right\}^{1 / q}=\left\|(I-A) \delta_{i}^{x}\right\|_{\ell^{q}(X)} \leq\|I-A\|<1-\frac{\epsilon}{2} . \\
\Rightarrow & \left\|a_{i i}^{x}\right\|_{X}>\frac{\epsilon}{2}, \quad \forall i \in \mathbb{N} .
\end{aligned}
$$

This contradicts that $\left\|a_{i j}^{x}\right\|_{X}<\frac{\epsilon}{2}, \forall i \geq j_{0}$.

Note: Theorems 3.1, 3.3 and 3.4 include the results of Hutton [9] given on p. 58-60 as particular case when $X$ is the field of scalars. 


\section{References}

[1] L. R. Acharya and M. Gupta, On Kolmogorov numbers of matrix transformations, Banach spaces and their applications in analysis, de Gruyter Proceedings in Mathematics, Berlin, New York (2007), 219-228.

[2] B. Carl and I. Stephani, Entropy, Compactness and the Approximation of Operators, Cambridge Univ. Press, Cambridge, 1990.

[3] P. Enflo, A counter example to the approximation problem in Banach spaces, Acta. Math., 130 (1973), 309317.

[4] N. De Grande - De Kimpe, Generalized sequence spaces, Bull. Soc. Math. Belgique 23 (1971), 123-166.

[5] M. Gupta and J. Patterson, The generalized $\ell^{p}$ spaces, Tamkang J. Math., 13 (1982), 161-179.

[6] _-, Matrix transformations on generalized sequence spaces, J. Math. Anal. Appl., 106 (1985), 54-68.

[7] A. Hinrichs, Approximation numbers of identity operators between symmetric sequence spaces, J. Approx. Theory 118 (2002), 305-315.

[8] A. Hinrichs and C. Michels, Approximation numbers of inclusions between Schatten classes, Rend. Circ. Mat. Palermo (2) Suppl. No. 76, (2005), 395-411.

[9] C. V. Hutton, J. S. Morrell and J. R. Retherford, Diagonal operators, approximation numbers and Kolmogoroff diameters, J. Approx. Theory 16 (1976), 48-80.

[10] J. A. Sampaio Martins, On the approximation numbers of embeddings of sequence spaces, Mathematical studies in honor of Professor Luis de Albuquerque (Portuguese), Univ. Coimbra, Coimbra, 1994.

[11] J. Patterson, Generalized sequence spaces and matrix transformations, Dissertation, I. I. T. Kanpur, India, 1980.

[12] A. Pietsch, Einige neue Klassen von kompakten linearen Abbildungen, Rev. Roumaine Math. Pures Appl. 8 (1963), 427-447.

[13] --, Nuclear Locally Convex Spaces, Springer-Verlag, Berlin, Heidelberg, New York, 1972.

[14] - - s- numbers of operators in Banach spaces, Studia Math., 51 (1974), 201-223.

[15] —-, Operator Ideals, VEB Deutscher Verlag der Wissenschaften, Berlin, 1978.

[16] —-, Eigenvalues and s-numbers, Cambridge University Press, New York, NY, USA, 1986.

[17] L. Skrzypczak, On approximation numbers of Sobolov embeddings of weighted sequence spaces, J. Approx. Theory 136 (2005), 91-107.

Department of Mathematics and Statistics, Indian Institute of Technology Kanpur, U.P.- 208016, India.

E-mail: manjul@iitk.ac.in

Department of Mathematics and Statistics, Indian Institute of Technology Kanpur, U.P.- 208016, India.

E-mail: lipi@iitk.ac.in 